\title{
Work Travel Mode Choice and Number of Non-Work Commute Stops
}

\author{
Chandra R. Bhat \\ Department of Civil and Environmental Engineering \\ University of Massachusetts at Amherst
}

\begin{abstract}
The research presented here develops a joint model of work mode choice and number of stops during the work commute. This model provides an improved basis to evaluate the effect of alternative policy actions to alleviate peak-period congestion. The model also contributes to activity-based research by allowing a more realistic behavioral representation of the simultaneous disaggregate choice process underlying mode choice to work and choice of number of activity stops. Mode choice is modeled using a multinomial logit model and number of stops is modeled using an ordered-response formulation. The joint model is applied to an empirical analysis using data from an activity survey conducted in the Boston Metropolitan area. The results underscore the importance of accommodating the inter-relationship between mode choice to work and number of activity stops in the work commute. The effects of a variety of congestion-alleviation measures are examined using the model.
\end{abstract}




\section{INTRODUCTION}

Mode choice modeling to work has been central to the evaluation of traffic congestion mitigation efforts which involve improvement in the level of service attributes of high occupancy travel modes (for example, designation of high-occupancy vehicle lanes on freeways) or disincentives to use the solo-auto mode (for example, congestion-pricing and additional gas taxes). The emphasis on work mode choice is a consequence of the morning and evening commute periods representing the most congested times of the weekday. Work mode choice models provide the tool to evaluate the ability of traffic-congestion actions to effect a change in mode of travel from solo-auto to high-occupancy vehicles.

In the past decade, however, transportation professionals have recognized that peak period congestion cannot be completely attributed to the home-to-work or work-to-home trip. Specifically, researchers have become aware of the substantial contribution (to traffic congestion) of the growing number of non-work trips made by individuals during the morning and evening commute (see Purvis, 1994 or Lockwood and Demetsky, 1994).

In this paper, we develop a joint model of work mode choice and number of non-work activity stops during the work commute (i.e., the total number of non-work stops made during the morning home-to-work commute and evening work-to-home commute). The joint model formulated here provides an improved basis to evaluate the effect on peak-period traffic congestion of conventional policy measures such as ridesharing improvements and solo-auto use disincentives. Traditional mode choice models address the question "What is the effect of a change in, say, solo-auto in-vehicle travel time (for example, due to conversion of an existing general-purpose lane to a high-occupancy lane) on work mode choice?" If commute trips were the sole contributors to peak period congestion, then the shifts in work mode choice provide a direct indication of the potential impact on congestion. A more pertinent question to address today, however, is "What is the effect of a change in, say again, solo-auto in-vehicle time on work mode choice and number of non-work stops?" This question is prompted by the recognition that vehicle trips due to non-work stops also add to peak period congestion. Thus, understanding the effect of a policy action on work mode choice and number of non-work stops allows us to evaluate the effect on peak-period congestion through the impact on both direct commute vehicle-trips and additional vehicle-trips due to non-work stops. 
Previous studies have not confronted the simultaneity in mode choice and number of nonwork stops during the work commute. They have focused on only one of these two choices. Some of these studies have considered one choice as being exogenous in the modeling of the other choice without recognizing the potential estimation problems arising from ignoring the simultaneity in the two choices. For example, Beggan (1988) examines work mode choice behavior and uses non-work stops as an exogenous variable in his model. Strathman et al. (1994), on the other hand, study trip chaining behavior during the work commute and use the mode used for the work trip as an explanatory variable. In addition, previous trip-chaining studies have been, for the most part, descriptive in nature as opposed to being policy sensitive (see Adiv, 1983; Hanson, 1980; Golob, 1986, and Strathman et al., 1994). The focus of these previous studies has been to examine the effect of household and personal characteristics on trip chaining behavior. While such studies are valuable in understanding the differential tendencies of households and individuals to chain trips, their value in policy analysis is limited since transportation policies have little impact on household and personal characteristics. This paper emphasizes the impact of both individual and household attributes and policy-relevant exogenous variables on stop-making behavior. Finally, most previous studies (including those mentioned above and the work by Damm, 1980 and Nishii et al., 1988) have modeled trip chaining as a binary choice of making no stops (simple work tour) or making one or more stops (complex tour); they have not examined the number of stops in a complex tour. ${ }^{1}$

The next section of this paper advances the econometric framework for the joint model system of mode choice and number of non-work stops. Section 3 discusses the data source and sample used in the empirical analysis. Section 4 focuses on empirical results. Section 5 examines the impact of policy actions using the model. The final section summarizes the important findings from the research.

\footnotetext{
${ }^{1}$ This paper, on the other hand, does not focus on spatial issues associated with destination choice of activity stops and does not go beyond trip chaining during the work tour. The reader is referred to O'Kelly and Miller (1984), Kitamura (1984), Goulias and Kitamura (1988) and Horowitz (1980) for studies of destination choice in the context of trip chaining, and to Adler and Ben-Akiva (1979), Pas (1984), Recker et al. (1987) and Hodge (1991) for the analysis of daily activity patterns.
} 


\section{ECONOMETRIC FRAMEWORK}

\subsection{Background}

The econometric framework models work mode choice and the number of non-work stops during the work commute jointly. Work mode choice is modeled using an unordered multinomial logit model and number of stops is modeled using an ordered-response formulation originally proposed by McElvey and Zavoina, 1975 (the author is not aware of any earlier effort to formulate and estimate such a joint unordered-ordered choice system in discrete-choice literature). The ordered-response formulation for number of stops recognizes the ordinal and discrete nature of stops.

The inter-relationship between mode choice and the choice of number of activity stops is accommodated by recognizing the endogeneity of the mode choice decision to the number of stops decision; that is, by recognizing the potential presence of self-selection in the mode choice decision based on the number of stops an individual wants to make (see Mannering and Hensher, 1987 for an extensive discussion of the self-selection problem). For example, an individual who chooses the solo-auto mode may have a higher propensity to make stops relative to another individual with the same observed characteristics but who does not choose solo-auto (this may arise, among other things, because individuals who are impulsive and who feel a strong need to be in control may prefer the solo-auto mode and, these same individuals, may also make many non-work stops). If we ignore this self-selection issue and model stop-making propensity using the subsample which chooses the solo-auto mode, then we run into an econometric bias problem because the expectation of the unobserved error component in the stop-making propensity equation is not zero, but some positive quantity.

A basic premise of the modeling system developed here is that the joint nature of mode choice to work and number of non-work stops arises because the two choices are caused or determined by certain common underlying observed and unobserved factors (see Train, 1986; page 85). For example, if the travel time by solo-auto is much less than transit, it may result in the choice of the solo-auto mode. Also, the low travel time by solo-auto may relax time constraints and lead to more stop-making during the work-commute. Thus, we will find a positive association between solo-auto mode choice and stop-making. We may also find a similar association because of positive correlation in unobserved factors that increase the choice of soloauto and increase stop-making. Thus, the reason for the joint nature of the two choices is because 
of common underlying factors, not because of direct causation between the choices. A different, but related, interpretation is that individuals choose a particular combination or "package" of mode choice and stops. Since both these choices are determined simultaneously, "it is not possible for one choice to cause the other, in a strict sense of causality" (Train, 1986; page 85). There is also a methodological rationale for adopting the current modeling approach. Maddala (1983) shows that there is a need for placing some restrictions in latent-variable model formulations involving discrete choices. Specifically, Maddala shows (see page 119 of his book) that it is logically inconsistent to have each choice affecting the other in a simultaneous latentvariable framework (the structural effect can only be in one direction). The interpretation that we adopt allows us to accommodate the joint nature of the two choices, while also being logically consistent from a methodological standpoint.

\subsection{Structure and Estimation}

In the following presentation, we will use the index $i$ to represent mode $(i=1,2, \ldots I)$, index $k$ to represent number of non-work stops $(k=0,1,2, \ldots K)$, and the index $q$ to represent the $q$ th individual $(q=1,2, \ldots Q)$. The equation system is then as follows:

$u_{q i}^{*}=\beta_{i}^{\prime} z_{q i}+\varepsilon_{q i}$, mode $i$ chosen if $u_{q i}^{*}>\max _{\substack{j=1,2, \ldots, I \\ j \neq i}} u_{q j}^{*}$

$s_{q i}^{*}=\gamma_{i}^{\prime} x_{q i}+\eta_{q i}, s_{q i}=k$ if $\delta_{i, k-1}<s_{q i}^{*} \leq \delta_{i, k}, s_{q i}$ observed for chosen mode $i$.

$u_{q i}^{*}$ is the indirect (latent) utility that the $q$ th individual derives from using the $i$ th mode, $s_{q i}^{*}$ is the (latent) stop-making propensity of the $q$ th individual should she or he use mode $i$, and $s_{q i}$ is the number of nonwork stops conditional on the choice of mode $i . s_{q i}$ is observed only for the chosen mode $i$ in the sample. $s_{q i}$ is characterized by the stop-making propensity $s_{q i}^{*}$ and the threshold bounds (the $\delta$ 's) in the usual ordered-response fashion. $z_{q i}$ and $x_{q i}$ are column vectors of exogenous variables specific to mode regime $i$, and $\beta_{i}$ and $\gamma_{i}$ are corresponding column vectors of parameters to be estimated. We assume that the $\varepsilon_{q i}$ values are identically and independently extreme-value distributed with a location parameter of zero across alternatives $i$ and individuals $q$. The $\eta_{q i}$ values are assumed to be identically normal-distributed across modes 
$i$ and individuals $q$ with a marginal standard distribution function $\Phi($.$) . The \eta_{q i}$ values are also assumed to be independent across individuals.

Let $R_{q i}$ be a dummy variable; $R_{q i}=1$ if the $i$ th mode is chosen by the $q$ th individual and $R_{q i}=0$ otherwise. Define

$v_{q i}=\left\{\max _{j=1,2, \ldots, I, j \neq i} u_{q j}^{*}\right\}-\varepsilon_{q i}$

The equation system in (1) can now be structured as:

$R_{q i}^{*}=\beta_{i}^{\prime} z_{q i}-v_{q i}, R_{q i}=1$ if $R_{q i}^{*}>0, R_{q i}=0$ otherwise

$s_{q i}^{*}=\gamma_{i}^{\prime} x_{q i}+\eta_{q i}, s_{q i}=k$ if $\delta_{i, k-1}<s_{q i}^{*} \leq \delta_{i, k}, s_{q i}$ observed for chosen mode $i$.

If there is a correlation between the random components $\left(v_{q i}, \eta_{q i}\right)$ for each mode, separate unidimensional mode choice and number of stops model estimations is not appropriate. The key to accommodating this correlation is to transform the random variable $v_{q i}$ into a standard normal random variable $v_{q i}^{*}$ as follows:

$v_{q i}^{*}=\Phi^{-1}\left[F_{i}\left(v_{q i}\right)\right]$,

where $\Phi($.$) is the standard normal distribution function and F_{i}$ is the multinomial logit distribution function of $v_{q i}$ implied by Equation (2) and the assumed iid extreme value distribution for the $\varepsilon_{q i}$ values. Now, since $\Phi\left(v_{q i}^{*}\right)=F_{i}\left(v_{q i}\right)$ by construction (see Equation 4), we can specify a bivariate distribution $L_{2}$ for $v_{q i}$ and $\eta_{q i}$ having the marginal distributions $F_{i}($.$) and$ $\Phi($.$) as (Lee, 1983):$

$L_{2}\left(v_{q i}, \eta_{q i}, \rho_{i}\right)=\Phi_{2}\left[\Phi^{-1}\left[F_{i}\left(v_{q i}\right)\right], \eta_{q i}, \rho_{i}\right]=\Phi_{2}\left(v_{q i}^{*}, \eta_{q i}, \rho_{i}\right)$,

where $\Phi_{2}($.$) denotes the bivariate normal distribution. From Equation (3) and the bivariate$ normal distribution of $v_{q i}^{*}$ and $\eta_{q i}$ (Equation 5), the joint probability of choosing mode $i$ and number of stops $k$ for individual $q$ is: 


$$
\begin{aligned}
& \begin{aligned}
P\left(R_{q i}=1, s_{q i}=k\right) & =\Phi_{2}\left[\Phi^{-1}\left\{F_{i}\left(\beta_{i}^{\prime} z_{q i}\right)\right\},\left(\delta_{i, k}-\gamma_{i}^{\prime} x_{q i}\right), \rho_{i}\right] \\
& -\Phi_{2}\left[\Phi^{-1}\left\{F_{i}\left(\beta_{i}^{\prime} z_{q i}\right)\right\},\left(\delta_{i, k-1}-\gamma_{i}^{\prime} x_{q i}\right), \rho_{i}\right] \text {, where }
\end{aligned} \\
& F_{i}\left(\beta_{i}^{\prime} z_{q i}\right)=\operatorname{Prob}\left(v_{q i}<\beta_{i}^{\prime} z_{q i}\right)=\frac{\exp \left(\beta_{i}^{\prime} z_{q i}\right)}{\sum_{j=1}^{I} \exp \left(\beta_{j}^{\prime} z_{q j}\right)}, i=1,2, \ldots, I .
\end{aligned}
$$

The parameters to be estimated in the joint model are the (K-1) $\delta_{i, k}\left(\delta_{i, 0}=-\infty\right.$ and $\left.\delta_{i, k}=+\infty\right)$ and the vectors $\beta_{i}$ and $\gamma_{i}$ for each mode $i$ (as structured, $x_{q i}$ does not include a constant). Defining a set of dummy variables

$$
M_{q k}=\left\{\begin{array}{l}
1 \text { if individual } q \text { makes } k \text { stops (irrespective of choice of mode) }(q=1,2, \ldots Q, k=1,2, \ldots K) \\
0 \text { otherwise, }
\end{array}\right.
$$

the log likelihood function for the estimation of the parameters in the model takes the form

$$
\log L=\sum_{q=1}^{Q} \sum_{i=1}^{I}\left\{R_{q i}\left(\sum_{k=1}^{K} M_{q k} \log \left[P\left(R_{q i}=1, s_{q i}=k\right)\right]\right)\right\}
$$

It is easy to see that if $\rho_{i}$ is equal to zero for each (and every) mode $i$, then the likelihood in Equation (9) partitions into a component corresponding to that of a discrete choice model for mode choice and another component which represents independent univariate ordered response models of number of stops for each mode. In general, ignoring $\rho_{i}$ and estimating independent models of non-work stops for each mode will lead to biased parameter estimates.

The maximization of the function in Equation (9) is achieved using a three-step procedure. In the first step, a discrete mode choice model is estimated along with independent ordered-response models of number of stops for each mode. In the second step, the discrete choice model parameters are held fixed and the log-likelihood function in Equation (9) is maximized with respect to the parameters in the number of stops model and the correlation parameters. Finally, the parameters from the second step are used as start values for the fullinformation maximum likelihood estimation of Equation (9). The likelihood function at each step is maximized using standard techniques. ${ }^{2}$

\footnotetext{
${ }^{2}$ Maximization is done using the GAUSS matrix programming language. The analytical gradients of the log-
} likelihood function with respect to the parameters are coded. 


\section{DATA SOURCE AND SAMPLE}

The data source used in the present study is a household activity survey conducted by the Central Transportation Planning Staff (CTPS) in the Boston Metropolitan region. The survey was conducted in April of 1991 and collected data on socio-demographic characteristics of the household and each individual in the household. The survey also included a one-day (mid-week working day) activity diary to be filled out by all members of the household above 5 years of age. Each activity pursued by an individual was described by: (a) start time, (b) stop time, (c) location of activity participation, (d) travel time from previous activity, (e) travel mode to activity location, and (f) activity type.

The sample for the current analysis comprises 618 employed adult individuals who made a work-trip on the diary day. The mode choice estimation is restricted to the choice of three modes due to data limitations and also because the remainder of the modes capture very little market share. The three modes are solo-auto (use of a car/van/pickup truck by one traveler), shared ride (use of a car/van/pickup truck by more than one traveler) and transit (bus, commuter rail, or local rail). The mode used for the final leg to work is used as the work mode choice (thus, if a person drops off another family member by car during the morning commute and then proceeds alone to work, the person's work mode choice is classified as solo-auto). The number of stops made during the work commute ranges from zero to four in the sample (dropping off/picking up individuals during the work commute is included as a stop except if it is part of a formal ridesharing arrangement among individuals of different households).

Level of service data were generated for each mode for each individual's trip to work These data were generated based on a combination of home location and work location information, manual reconstruction of most likely path for non-chosen modes, estimated times from an interim regional model for solo-auto and shared-ride in-vehicle travel, estimated times from published transit schedules for the transit mode, and estimated parking, access/egress, and line-haul costs. A detailed description of the actual procedures and assumptions is beyond the scope of the current paper, but is available in Gallagher (1993).

The sample is choice-based with respect to mode choice and has been weighted in an attempt to make it representative of the market work mode shares as reflected in the 1990 Census Journey-to-Work results for the Boston Metropolitan area. The Weighted Exogenous Sample 
Maximum Likelihood (WESML) method proposed by Manski and Lerman (1977) is used in estimation. The asymptotic covariance matrix of parameters is computed as $H^{-1} \Delta H^{-1}$, where $H$ is the hessian and $\Delta$ is the cross-product matrix of the gradients ( $H$ and $\Delta$ are evaluated at the estimated parameter values).

The mode share in the (weighted) sample is as follows: $76.55 \%$ solo-auto, $11.31 \%$ shared-ride and $12.14 \%$ transit. About $72 \%$ of individuals in the sample make no stops during the work tour, while $28 \%$ make one or more stops. Among individuals who drive alone, $70 \%$ make no stops and the remainder make one or more stops. The corresponding figures among individuals who share a ride and who take transit are $76 \%$ and $80 \%$, respectively. These figures suggest that mode choice and stop-making are inter-dependent.

\section{EMPIRICAL ANALYSIS}

\subsection{Model Specification}

The choice of variables for potential inclusion in the model was guided by previous theoretical and empirical work on mode choice modeling and trip chaining analysis, and intuitive arguments regarding the effects of exogenous variables. We arrived at the final specification based on a systematic process of eliminating variables found to be statistically insignificant in previous specifications and based on considerations of parsimony in representation. Some variables with marginally significant coefficients are retained in the final specification, either for the sake of completeness or because they provide useful and suggestive insights. Tables $1 \mathrm{a}$ and $1 \mathrm{~b}$ provide a list of exogenous variables used in the model, their definitions, and associated descriptive statistics in the sample.

We constrained the parameters on all non-level of service variables to be equal across the different mode regimes for the stop-making propensity equation. We adopted this specification because we did not have any strong theoretical reason to believe that the effect (on stop-making propensity) of socio-demographic variables, the household total number of stops variable, and the work time variable should be different for different modes. Further, constraining parameters enhances the stability of the model and preserves degrees of freedom. We tested for different parameters on the level-of-service variables in the stop-making propensity equation for the different mode regimes (the effect of the level-of-service variables may differ based on mode since, for example, travel time by bus may be more tiring and may have a larger negative effect 
on stop-making propensity than travel time by car). However, a statistical likelihood ratio test of equality of the effect of level-of-service parameters on stop-making propensity across the mode regimes could not be rejected. Hence, we maintained equal parameters on all exogenous variables in the different mode regimes.

Finally, we tested two different specifications for the thresholds (the $\delta_{i k}$ parameters in Equation 1) that determine the correspondence between the latent stop-making propensity and the actual number of stops in the ordered response formulation. The first specification allowed the thresholds to be completely unconstrained across the mode regimes (there are 4 thresholds in each mode regime and, since there are three modes, the total number of thresholds in this specification is 12). The second specification restricted the thresholds to be the same for each mode regime up to a structural shift (that is, all thresholds for each mode regime are shifted by the same amount relative to the corresponding threshold in a base mode regime; the total number of parameters characterizing the thresholds in this specification is six, four thresholds in the base mode regime and two structural shift terms for the two remaining mode regimes). This second specification is equivalent to maintaining the same thresholds for all mode regimes, but introducing constants in the stop propensity equations in two of the three mode regimes. A likelihood ratio test between the two specifications did not reject the second specification relative to the first (the test statistic was 5.66, which is smaller than the chi-squared critical value with 6 degrees of freedom at any reasonable level of significance). Hence we used the second specification for the thresholds in the current analysis.

\subsection{Overall Empirical Results}

The log-likelihood value at convergence for the joint model system is -792.1. The log-likelihood when only alternative specific constants are included in the mode choice model and when only the threshold parameters are introduced in the number of stops model is -980.0. A log-likelihood ratio test clearly rejects the null hypothesis that all exogenous variable parameters and error correlations are zero. A further likelihood ratio test of the joint model with an independent model (where all the correlation terms are set to zero) rejects the hypothesis that mode choice and number of stops are independently determined (the log-likelihood value of the independent model is -796.65; the likelihood ratio value for the test is 9.1 which is larger than the chi-squared statistic of 7.82 with 3 degrees of freedom at the 0.05 level). 
The next three sections of the paper present the results of the multinomial mode choice model, the ordered number of stops model, and the error correlation parameter estimates, respectively. It should be noted that the exogenous variable parameters in the different models and those of the error correlations are all estimated simultaneously. We discuss them separately for ease in presentation.

\subsection{Mode Choice Model}

Table 2 presents the results of the mode choice model. The effects of the socio-demographic variables are as expected. Individuals with high income tend to prefer the solo-auto mode over other modes and prefer the shared-ride mode over the transit mode. As the ratio of the number of vehicles to workers in a household increases, there is less competition for cars among household members and hence a lower tendency to use the shared-ride mode and an even lower likelihood of using the transit mode.

The level-of-service measures yield reasonable parameters (an earlier specification that deflated travel cost by income was found to be less satisfactory than the cost specification adopted here). The implied cost of in-vehicle travel time is $\$ 7.30$ per hour and that of out-ofvehicle time is $\$ 11.95$ per hour (computed at the mean one-way travel distance to work of 9.74 miles).

The duration at work has a positive effect on solo-auto utility, presumably because a high work duration results in increased time constraints and more preference for a fast mode. A high employment density may result in more stressful driving (because of stop-and-go traffic); this may explain the shift away from the solo-auto mode.

Finally, a note on the transit mode constant. The high magnitude of this constant reflects factors that explain the choice of transit for some people despite high transit travel times (for example, in a two-worker household with one car, one of the workers may be captive to transit; however, the current model considers this worker to have all three modes available).

\subsection{Number of Stops Model}

The parameter estimates of the number of stops model are shown in Table 3. The positive influence of income on stop-making propensity is consistent with the results from earlier studies (see Strathman et al., 1994; Goulias and Kitamura, 1989). The effect of age indicates that older 
people are less inclined to make stops during the work commute, possibly because of their lower activity levels. Individuals who live alone or who live in a couple family household have less familial responsibilities than other household types (nuclear family, single parent, returning young-adult and other extended family households) and hence can engage in more activities during the return home, as the results suggest. We did not find any gender-based differences for unmarried individuals; however, married women were more likely to make stops than married men. This may be a reflection of the continuing trend of women to be primarily responsible for household maintenance activities and for dropping/picking up children from day-care (see Mensah, 1995). The effect of young children is represented by the final two socio-demographic variables. In households with young children and with at least one unemployed adult, the employed adult makes fewer stops than employed adults in other non-single and non-couple households. This might be due to an altruistic motive to provide "relief" to the other adult caregiver and also due to a desire to spend time with the child (or children) and the family after a long day away from home. However, when all the adults are employed in a household with young children, the employed adults have to make additional stops to pick up/drop off their kids at the day-care, as suggested by the positive sign on the final socio-demographic variable. The net stop-making propensity of adults in such households is not significantly different from those in other non-single and non-couple households.

The level-of-service of travel between home and work affects number of stops during the work commute (note that the level-of-service is a function of mode chosen; in the current model, this endogeneity of the level-of-service variables based on mode choice is incorporated through the accommodation of self-selection in mode choice). A higher travel time to work results in tighter time constraints and consequently appears to reduce number of stops made by individuals. The negative coefficient on the out-of-vehicle travel time over distance variable suggests that out-of-vehicle time is perceived as being more onerous and tiring (particularly when the distances traveled are short) than in-vehicle time, leading to a greater reduction in stop-making propensity (the effects of the travel time variables were smaller in magnitude and statistically insignificant when the endogeneity of the mode choice decision was ignored). Travel cost to work had a very insignificant impact on stop-making propensity. Since we also did not have any strong theoretical reason to expect travel cost to affect non-work stops, we did not include it in the specification. 
The duration at work has a negative effect on stop-making propensity, presumably again because of the resulting higher time constraints. Finally, the total number of non-work stops made by the household (a proxy for household activity demand level; see Strathman et al., 1994) has, as expected, a positive impact on stop-making propensity of individuals in that household. We did not find any significant effect of work schedule (arrival time at work and departure time from work) on stop-making propensity. This suggests that, in the context of the current analysis, time of travel does not impact stop-making propensity.

\subsection{Correlation Parameters}

The joint modeling of mode choice and number of stops during the work tour is necessitated by the potential presence of correlation in unobserved elements affecting the two decisions. The results (see Table 4) indicate statistically significant correlations between unobserved factors affecting transit and solo-auto mode utilities and stop-making propensity (the reader will note that we arrived at the same conclusion using a log-likelihood ratio test in Section 4.2).

The parameters in Table 4 represent the correlation between the error terms $v_{q i}$ and $\eta_{q i}$. Thus, a positive parameter indicates a negative correlation between unobserved factors affecting the corresponding mode utility and stop-making propensity, while a negative parameter implies a positive correlation (see Equation 3). Our results show that unobserved factors (say, need for control and independence, impulsiveness, etc.) that increase the preference for the solo-auto mode (and to a lesser extent, for the transit mode) also increase stop-making propensity, while unobserved factors (say, low activity levels) that increase the preference for the shared-ride mode decrease stop-making propensity. As we will see in the next section, these correlations have substantial implications for policy analysis.

\section{POLICY IMPLICATIONS}

The model developed in this paper can be used to examine a wide variety of policy actions. Most transportation congestion management actions attempt to effect a change in mode choice or reduce trip-making during the peak period by directly or indirectly impacting the level-of-service variables. For example, congestion-pricing and parking-pricing schemes rely on the use of monetary disincentives for use of the solo-auto mode, while an employer-based trip-reduction plan that aims at making solo-auto parking far removed from the work place increases solo-auto 
out-of-vehicle travel time. Improvements to transit service may involve more frequent service and more extensive route coverage (thereby decreasing transit out-of-vehicle travel time by reducing wait time and walking time, respectively), or introduction of additional express services (thereby reducing in-vehicle travel time). Employer-based monetary incentives may subsidize transit fares or lower high-occupancy vehicle parking costs. Conversion of existing general lanes to high-occupancy vehicle (HOV) use lanes on expressways increases in-vehicle travel time by solo-auto, while decreasing in-vehicle travel times by high-occupancy vehicles. The implementation of all of these policies and others that attempt to alleviate congestion by reducing work duration can be reflected through changes in the appropriate exogenous variables in the joint model system.

Conventional mode choice models only consider the potential impact on work trips due to policy actions; they are unable to estimate the impacts on non-work trips during the peak period. Since non-work trips also contribute to peak period congestion, conventional mode choice models do not provide adequate information on the effectiveness of alternative congestion-alleviation actions. An alternative approach to evaluation of congestion-alleviation actions may involve the development of independent mode choice and number of stops models. The joint choice probability of each mode-number of stops combination can then be obtained by multiplying the estimated probabilities of each individual choice. However, as indicated earlier, this approach ignores the correlation in unobserved factors that affect both mode choice and number of stops (i.e., it ignores the joint nature of the two decisions). Consequently, this alternative approach can also provide misleading projections of the impact of policy actions.

In the rest of this section, we examine the impacts of changes in policy-relevant exogenous variables on number of stops by the solo-auto mode. The reader will note that policy analysts may also be interested in the impact of policy actions on use of other non solo-auto modes. While this can be studied using the joint model, we focus our attention on solo-auto trips in this paper for the purpose of limiting the discussion and also because solo-auto trips contribute most to traffic congestion. We first compare the results of the effects of exogenous variables on solo-auto stops obtained using the independent mode choice and number of stops model (independence model) and the joint mode choice and number of stops model (joint model). We then examine the impact of alternative policy actions on total solo-auto trips using the joint model. 
The impact of policy actions on solo-auto stops can be evaluated by modifying exogenous variables to reflect a change, computing revised disaggregate probabilities, calculating revised expected aggregate values for number of stops by the solo-auto mode, and then obtaining a percentage change from the baseline estimates.

Table 5 provides the estimated percentage change (at the aggregate level) in each category of non-work stops for the solo-auto mode in response to a five minute decrease (on average across the sample) in in-vehicle and out-of-vehicle travel times for the transit and shared-ride modes (note that since some individuals have a current in-vehicle/out-of-vehicle time of less than five minutes, it is not possible to decrease travel time by five minutes on an individual basis and hence the scenario of a five minute decrease on average). The five minute decrease (on average) corresponds to about a 17\% (21\%) decrease in transit (shared-ride) invehicle time and a 16\% (65\%) decrease in transit (shared-ride) out-of-vehicle travel time for each individual in the sample. The results indicate that the independence model shows an almost equal percentage decrease in all the stops categories for all transit and shared-ride service improvements. However, the joint model shows that an improvement in transit service draws solo-auto drivers very unevenly based on the number of stops. The largest draw is from solo-auto drivers who do not make any stops and the smallest draw is from solo-auto drivers who make four stops. This is a consequence of the significant and large positive correlation between the solo-auto utility and stop-making propensity; individuals who make many stops are unlikely to be drawn away from solo-auto. Another expected result from both the independence and joint models in the table is that out-of-vehicle time improvements result in larger decreases in soloauto use for each stop category than equivalent (absolute-value) in-vehicle time improvements.

The net percentage change in total number of stops made by the solo-auto mode can be computed as:

Net \% change in total stops by solo-auto mode $=\sum_{k=0}^{K}\left(\frac{k n_{a k}}{\sum_{k} k n_{a k}}\right) \zeta_{a k}$

where $n_{a k}$ is the expected number of individuals who use the solo-auto mode and make a work tour characterized by $k$ stops and $\zeta_{a k}$ is the percentage aggregate change in each stop category for the solo-auto mode. This overall effect on number of stops is shown in the final column of Table 5. The independence model projects a substantially more optimistic view of the reduction 
in solo-auto stops than the joint model; in particular, the independence model overestimates the percentage reduction in solo-auto stops by more than $100 \%$ for every policy scenario (we found a similar difference in results between the independence and joint models for solo-auto use disincentives and for work schedule changes). Since each non-work stop contributes an additional trip to peak-period congestion, this implies that the independence model provides an overly optimistic projection of reduction in peak-period congestion.

\section{SUMMARY AND CONCLUSIONS}

In this paper, we have developed a joint model of work mode choice and number of non-work activity stops during the work commute. The methodology developed here represents, to the author's knowledge, the first attempt in discrete choice literature to formulate and estimate a model with an unordered multinomial choice as well as an ordered multinomial choice.

The empirical analysis uses a data set from the Boston Metropolitan area. The results indicate the strong effects of level-of-service measures to work, income, number of vehicles per worker in the household, work duration, and employment density at the work place on mode choice to work. Socio-economic variables, work duration, and the overall activity level of the household (proxied by total household non-work stops) significantly influence stop-making propensity during the work commute. In-vehicle and out-of-vehicle travel times to work negatively influence stop-making propensity, but we did not find any significant effect of travel cost to work on stop-making propensity. Our empirical analysis also shows strong correlations in random components influencing mode choice and stop-making propensity. These correlations have a substantial impact on the aggregate percentage change in each stop category in response to changes in policy-relevant exogenous variables. The joint model shows that non solo-auto mode improvements and auto-use disincentives draw little share of solo-auto drivers who make many stops and draw a high share of solo-auto drivers who do not make any stop.

A limitation of the current joint model from a policy perspective is that it does not consider substitution effects between non-work stops during the work commute and non-work stops made after returning back home in the evening. In particular, a reduction in non-work stops during the work commute (in response to some policy action) may result in increased participation in out-of-home activities after returning home. If some of these participations are pursued during the peak commuting hour, the reduction in solo-auto trips due to the reduction in 
commute stops may be just replaced by additional solo-auto trips after arriving home. While there is some empirical evidence to suggest the presence of such substitution effects (see Hamed and Mannering, 1993), we found that trips after arriving home from work tend to be made beyond the peak commuting period (at least in the current sample) and thus do not contribute substantially to peak congestion.

\section{Acknowledgements}

This research is part of an ongoing NSF-funded research project titled "Measurement, Modeling, and Prediction for Infrastructure Systems" and awarded to the National Institute of Statistical Sciences (NISS). The comments of Prof. Eric Pas, Dr. Alan Karr, and Dr. Agostino Nobile during a presentation of the initial results of this research at NISS are greatly appreciated. The author would also like to thank Mr. Jim Gallagher, Mr. Ian Harrington, and Ms. Julie McLennan of the Central Planning Transportation Staff in Boston for providing the data and for clarifying data issues. Frank Koppelman, Keith Lawton, and several anonymous referees provided valuable comments and suggestions on an earlier draft of the paper. 


\section{References}

Adiv, A. (1983). The structure of work-trip based on analysis of trip diaries in the San Francisco Bay area. In Recent Advances in Travel Demand Analysis, S. Carpenter and P. Jones (eds.), 335-349, Gower, Aldershot, U.K.

Adler, T. and M.E. Ben-Akiva (1979). A theoretical and empirical model of trip chaining behavior. Transportation Research, 13B, 243-257.

Beggan, J.G. (1988). The Relationship Between Travel/Activity Behavior and Mode Choice for the Work Trip. Unpublished Master's Thesis, Northwestern University.

Damm, D. (1980). Interdependencies in activity behavior. Transportation Research Record, 750, 33-40.

Feller, W. (1971). An Introduction to Probability Theory and its Applications. Wiley, New York.

Gallagher, J. (1993). Development of regional interim home-based work mode choice models. Technical Memorandum, May 14.

Golob, T.F. (1986). A non-linear canonical correlation analysis of weekly chaining behavior. Transportation Research, 20A, 385-399.

Goulias, K.G. and R. Kitamura (1988). On the spatial distribution of linked trips in a uniform two-dimensional space. Unpublished report, Department of Civil Engineering, University of California, Davis, CA.

Goulias, K.G. and R. Kitamura (1989). Recursive model system for trip generation and trip chaining. Transportation Research Record, 1236, 59-66.

Hamed, M.M and F.L. Mannering (1993). Modeling travelers' postwork activity involvement: toward a new methodology. Transportation Science, 27(4), 381-394.

Hanson, S. (1980). The importance of multipurpose journey to work in urban travel behavior. Transportation, 9, 229-248.

Hodge, D.C. (1991). Development of methods of analysis for planning transit system components in and around major activity centers, Part I: Trip chaining: the behavioral basis for the design of circulation systems for major activity centers. Final report, Transport Northwest regional Center, Seattle, University of Washington.

Horowitz, J.L. (1980). A utility maximizing model of the demand for multi-destination non-work travel. Transportation Research, 14B, 369-386.

Kitamura, R. (1984). Incorporating trip chaining into analysis of destination choice. Transportation Research, 18B, 67-81.

Lee, L.F. (1983). Generalized econometric models with selectivity. Econometrica, 51(2), 507512.

Lockwood, P.B. and M.J. Demetsky (1994). Nonwork travel - A study of changing behavior. Presented at the 73rd Annual Meeting of the Transportation Research Board, Washington, D.C., January.

Maddala, G.S. (1983). Limited Dependent and Qualitative Variables in Econometrics. Cambridge University Press, Cambridge. 
Mannering, F.L. and D.A. Hensher (1987). Discrete/Continuous econometric models and their application to transport analysis. Transport Reviews, 7(3), 227-244.

Manski, C. and S. Lerman (1977). The estimation of choice probabilities from choice-based samples. Econometrica, 45, 1977-1988.

McKelvey, R.D. and Zavoina, W. (1975). A statistical model for the analysis of ordinal level dependent variables. Journal of Mathematical Sociology, 4, 103-120.

Mensah, J. (1995). Journey to work and job characteristics of the urban poor: A gender analysis of a survey data from Edmonton, Alberta. Transportation, 22, 1-19.

Nishii, K., K. Kondo and R. Kitamura (1988). An empirical analysis of trip chaining behavior. Presented at the 67th Annual Meeting of the Transportation Research Board, Washington, D.C., January.

O'Kelly, M.E. and E.J. Miller (1984). Characteristics of multistop multipurpose travel: an empirical study of trip length. Transportation Research Record, 976, 33-39.

Pas, E.I. (1984). The effect of selected sociodemographic characteristics on daily travel-activity behavior. Environment and Planning, 16A, 571-581.

Purvis, C.L. (1994). Changes in regional travel characteristics and travel time budgets in the San Francisco bay area: 1960-1990. Presented at the 73rd Annual Meeting of the Transportation Research Board, Washington, D.C., January.

Recker, W.W., M.G. McNally, and G.S. Root (1987). An empirical analysis of urban activity patterns. Geographic Analysis, 19, 166-181.

Strathman, J.G., K.J. Dueker and J.S. Davis (1994). Effects of household structure and selected travel characteristics on trip chaining. Transportation, 21, 23-45.

Train, K. (1986). Qualitative Choice Analysis: Theory, Econometrics, and an Application to Automobile Demand. The MIT Press, Cambridge, Massachusetts. 


\section{LIST OF TABLES}

Table 1a. Socio-demographic and other non-level of service variable definitions and sample statistics $(n=618)$

Table 1b. Level-of-service variables and associated sample statistics $(n=618)$

Table 2. Mode choice model estimates

Table 3. Number of stops (in work commute) model

Table 4. Estimates of correlation between mode choice utility and stop-making propensity

Table 5. Impact of policy actions on number of solo-auto stops 
Table 1a. Socio-demographic and other non-level of service variable definitions and sample statistics $(n=618)$

\begin{tabular}{|c|c|c|c|}
\hline \multirow{2}{*}{ Variable } & \multirow{2}{*}{ Definition } & \multicolumn{2}{|c|}{ Sample Statistics } \\
\hline & & Mean & SD \\
\hline \multicolumn{4}{|l|}{ Socio-demographic } \\
\hline Income & Annual household income in $\$ 0000$ s of dollars & 6.19 & 2.75 \\
\hline Age & Age of individual in years $\left(\times 10^{-1}\right)$ & 4.18 & 1.28 \\
\hline Vehicles per worker in household & Number of vehicles per worker in individual's household & 1.00 & 0.49 \\
\hline Single individual household & 1 if individual lives alone & 0.13 & 0.34 \\
\hline Couple household & 1 if individual lives with another adult; the two adults may or may not be married & 0.35 & 0.48 \\
\hline Female and married & 1 if individual is a woman and is married & 0.37 & 0.48 \\
\hline Presence of kids $<11 \mathrm{yr}$ & 1 if there are one or more children less than $11 \mathrm{yr}$ in individual's household & 0.19 & 0.40 \\
\hline $\begin{array}{l}\text { Presence of kids }<11 \text { yr and no } \\
\text { unemployed adults } \\
\text { Other variables }\end{array}$ & $\begin{array}{l}1 \text { if there are one or more children less than } 11 \mathrm{yr} \text { and there are no unemployed adults in } \\
\text { individual's household }\end{array}$ & 0.10 & 0.30 \\
\hline Work duration & Work duration (in 100s of min) & 5.15 & 1.07 \\
\hline Employment density at work zone & Employment density at the zone of individual's employment $\left(\times 10^{-2}\right)$ & 1.69 & 3.06 \\
\hline Total household number of stops & Total number of non-work stops made by all individuals in the household in the day & 1.23 & 1.42 \\
\hline
\end{tabular}

Table 1b. Level-of-service variables and associated sample statistics $(n=618)$

\begin{tabular}{|c|c|c|c|c|c|c|}
\hline \multirow{2}{*}{ Variable (all variables refer to one-way travel) } & \multicolumn{2}{|c|}{ For solo-auto } & \multicolumn{2}{|c|}{ For shared-ride } & \multicolumn{2}{|c|}{ For transit } \\
\hline & Mean & SD & Mean & SD & Mean & SD \\
\hline Total travel time to work (in min) & 25.40 & 12.70 & 31.10 & 13.99 & 60.24 & 36.12 \\
\hline Out-of-vehicle travel time over distance to work (in min/mile) & 1.31 & 1.47 & 1.78 & 1.91 & 5.47 & 5.03 \\
\hline Travel cost to work (\$) & 2.09 & 1.90 & 1.04 & 0.95 & 1.84 & 1.59 \\
\hline
\end{tabular}


Table 2. Mode choice model estimates

\begin{tabular}{|c|c|c|}
\hline Variable & Coefficient & $t$-statistic \\
\hline \multicolumn{3}{|l|}{ Mode constants (solo-auto is base) } \\
\hline Shared-ride & 0.290 & 0.40 \\
\hline Transit & 3.823 & 4.85 \\
\hline \multicolumn{3}{|l|}{ Socio-economic characteristics (solo-auto is base) } \\
\hline \multicolumn{3}{|l|}{ Income - in $\$ 0000 / y r$} \\
\hline Shared-ride & -0.021 & -0.42 \\
\hline Transit & -0.108 & -1.93 \\
\hline \multicolumn{3}{|l|}{ Vehicles per worker in household } \\
\hline Shared-ride & -0.865 & -2.20 \\
\hline Transit & -1.235 & -3.95 \\
\hline \multicolumn{3}{|l|}{ Level-of-service measures (generic) } \\
\hline Total travel time to work - in min & -0.054 & -6.54 \\
\hline Out-of-vehicle travel time over distance to work - in min/mile & -0.336 & -5.62 \\
\hline Total travel cost to work - in \$ & -0.442 & -3.06 \\
\hline \multicolumn{3}{|l|}{ Work duration and location variables (specific to solo-auto) } \\
\hline Work duration - in 100s of min & 0.215 & 2.27 \\
\hline Employment density at work zone & -0.074 & -1.28 \\
\hline
\end{tabular}

Table 3. Number of stops (in work commute) model

\begin{tabular}{|l|c|c||}
\hline Variable & Coefficient & $\boldsymbol{t}$-statistic \\
\hline Constant (in shared-ride regime) & -0.094 & 8.63 \\
Constant (in transit regime) & 0.706 & 5.18 \\
Socio-economic characteristics & & \\
$\quad$ Income - in \$0000/yr & 0.072 & 2.65 \\
Age & -0.129 & -2.66 \\
Single individual households & 0.541 & 2.22 \\
Couple household & 0.534 & 3.28 \\
Female and married & 0.320 & 2.16 \\
Presence of kids < 11 yr & -0.341 & -1.15 \\
Presence of kids <11 yr and no unemployed adults & 0.386 & 1.18 \\
Level-of-service measures & & \\
Total travel time to work - in min & -0.012 & 2.72 \\
Out-of-vehicle travel time over distance to work - in min/mile & -0.553 & -3.95 \\
Work duration and household activity level variables & & \\
Work duration - in 100s of min & -0.316 & -5.37 \\
Total number of non-work stops made by household & 0.247 & 5.34 \\
Threshold propensity demarcating & & \\
$\quad$ zero and one stop & -0.703 & -1.62 \\
one and two stops & 0.047 & 0.11 \\
two and three stops & 0.682 & 1.90 \\
three and four stops & 1.225 & 2.80 \\
\hline \hline
\end{tabular}


Table 4. Estimates of correlation between mode choice utility and stop-making propensity

\begin{tabular}{||l|c|c||}
\hline Correlation in errors between... & Estimate & $\boldsymbol{t}$-statistic \\
\hline \hline Transit utility and stop-making propensity equations & -0.440 & -2.40 \\
Shared-ride utility and stop-making propensity equations & 0.343 & 1.02 \\
Solo-auto utility and stop-making propensity equations & -0.655 & -2.34 \\
\hline
\end{tabular}

Table 5. Impact of policy actions on number of solo-auto stops

\begin{tabular}{||l||c|c|c|c|c|c|c||}
\hline \hline \multirow{2}{*}{ Policy scenario } & \multicolumn{3}{|c|}{ Percentage aggregate change in solo-auto stops during the work commute } \\
\hline $\begin{array}{l}\text { Five minute reduction } \\
\text { (on average) in... }\end{array}$ & Model & $\mathbf{0}$ stops & $\mathbf{1}$ stop & $\mathbf{2}$ stops & $\mathbf{3}$ stops & $\mathbf{4}$ stops & $\begin{array}{c}\text { Net } \\
\text { effect }\end{array}$ \\
\hline \hline $\begin{array}{l}\text { Transit in-vehicle travel } \\
\text { time }\end{array}$ & Independent & -1.402 & -1.369 & -1.322 & -1.284 & -1.273 & -1.326 \\
& Joint & -1.648 & -0.846 & -0.634 & -0.524 & -0.472 & -0.671 \\
\hline $\begin{array}{l}\text { Shared-ride in-vehicle } \\
\text { travel time }\end{array}$ & Independent & -3.882 & -4.071 & -4.016 & -3.884 & -3.646 & -3.965 \\
& Joint & -5.016 & -1.725 & -0.926 & -0.512 & -0.258 & -1.060 \\
\hline Transit out-of-vehicle \\
travel time & Independent & -3.514 & -3.665 & -3.697 & -3.709 & -3.716 & -3.690 \\
\hline $\begin{array}{l}\text { Shared-ride out-of-vehicle } \\
\text { travel time }\end{array}$ & Joint & -4.256 & -2.311 & -1.717 & -1.278 & -0.878 & -1.748 \\
\hline & Independent & -7.661 & -7.784 & -7.664 & -7.532 & -7.422 & -7.654 \\
& Joint & -9.688 & -3.516 & -2.124 & -1.320 & -0.721 & -2.310 \\
\hline
\end{tabular}

